\title{
Two Overlapped Coins Impacted in Cricopharynx: A Case Report
}

\author{
Anshul Singh ${ }^{1}$, Manish Gupta ${ }^{2 *}$ and Akanksha Gupta ${ }^{3}$ \\ ${ }^{1}$ Senior Resident, Maharishi Markandeshwar Institute of Medical Sciences and \\ Research, MMDU, Ambala, India \\ ${ }^{2}$ Professor and Head, Department of ENT, Maharishi Markandeshwar Institute of \\ Medical Sciences and Research, MMDU, Ambala, India \\ ${ }^{3}$ MBBS Student, School of Medical Science and Research, Sharda University, Greater \\ Noida, India \\ *Corresponding Author: Manish Gupta, Professor and Head, Department of ENT, \\ Maharishi Markandeshwar Institute of Medical Sciences and Research, MMDU, \\ Ambala, India.
}

Received: October 14, 2021

Published: October 04, 2021

(C) All rights are reserved by Manish Gupta., et al.

\begin{abstract}
Foreign body in ear, nose and throat is commonly encountered in otorhinolaryngology practice. Among which coin in the throat is the common foreign body seen but rarely multiple coin swallowing may occur and if present they may get impacted at different locations. We report a 10-year-old with history of coin ingestion but detailed analysis of x-ray and retrospective history confirmed it to be multiple coins. The emergency rigid esophagoscopy was undertaken and 2 coins impacted at the same place were successfully removed.
\end{abstract}

Keywords: Esophageal Foreign Body; Esophagus; Esophagoscopy; Cricopharynx; Roentgenogram

\section{Introduction}

Children, due to curiosity, swallow a variety of foreign bodies and coin is the commonest one [1]. Coin once swallowed gets lodged at the cricopharynx because of its narrow diameter [1-3]. Coins are smooth, round and thin and thus get easily swallowed by children. However, when impacted may cause obstruction and may be even life threatening in absence of early intervention [4].

Young children, because of their habit to explore new objects in their contact and tendency of putting it in their mouth, are more often present with foreign bodies in their throat [3]. Also, the neuromuscular coordination is poor in young children, leading to accidental aspiration or swallowing of foreign bodies. In older children, the esophagus foreign body is seldom seen, because they wouldn't ingest something inadequate or small coin passes through [3].

\section{Case Presentation}

A 10-year-old boy came to our emergency with a history of accidental coin ingestion, one day back. The mother of the child informed about a single coin being swallowed. On clinical examination the child was sitting comfortably with no breathing difficulty, cough, pain or drooling of saliva. Roentgenogram antero-posterior and lateral view neck showed a radio-opaque foreign body, round in shape in front of C6-C7 cervical vertebrae (Figure 1 and 2). On close examination of lateral view, the round shadow of anterior and posterior edges was at different levels, suggestive of the possibility of multiple coins or a button battery, which is also commonly ingested by children. On taking detailed history, the child admitted that he had ingested two coins simultaneously. The patient was taken up for emergency rigid esophagoscopy in general anesthesia, 
for foreign body removal. Intra-operatively, two coins, lying at the same place, overlapping and sticking to each other were seen in cricopharynx. Both the coins (Figure 3) were caught with alligator forceps and removed simultaneously. The intra and postoperative periods were uneventful.

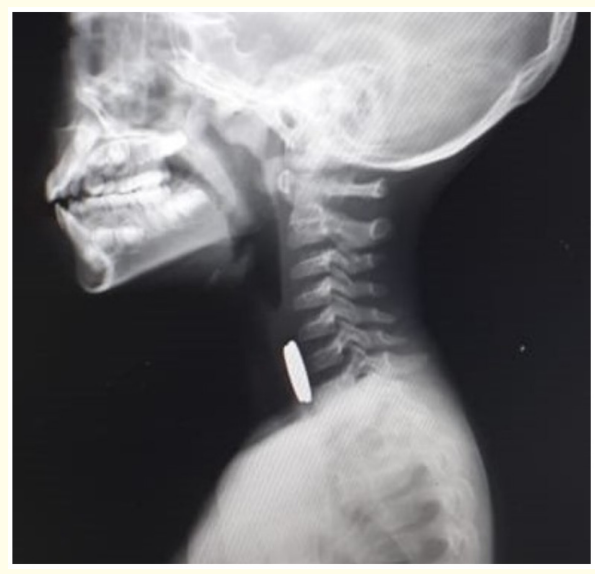

Figure 1: The soft tissue $x$-ray neck lateral view showing a radio opaque foreign body, at the level of C6-C7 cervical vertebrae. Note the anterior and posterior margins are not at the same level.

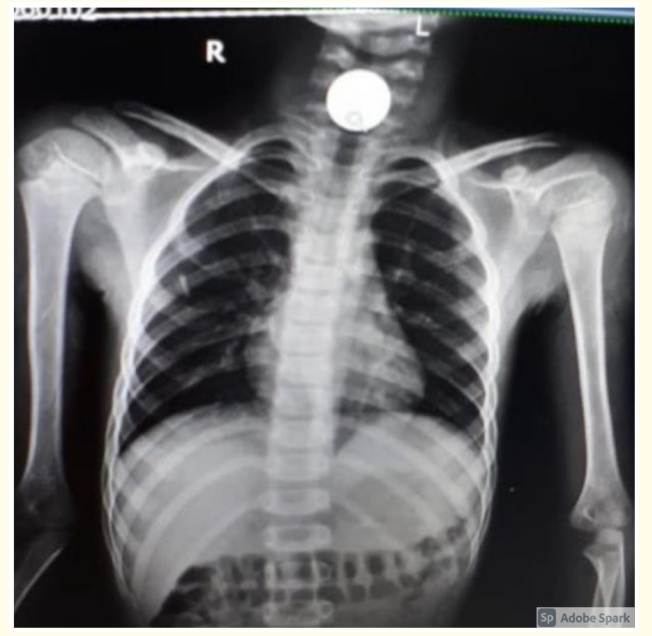

Figure 2: The AP view $x$-ray of neck and chest showing a circular radio opaque foreign body above the clavicle.

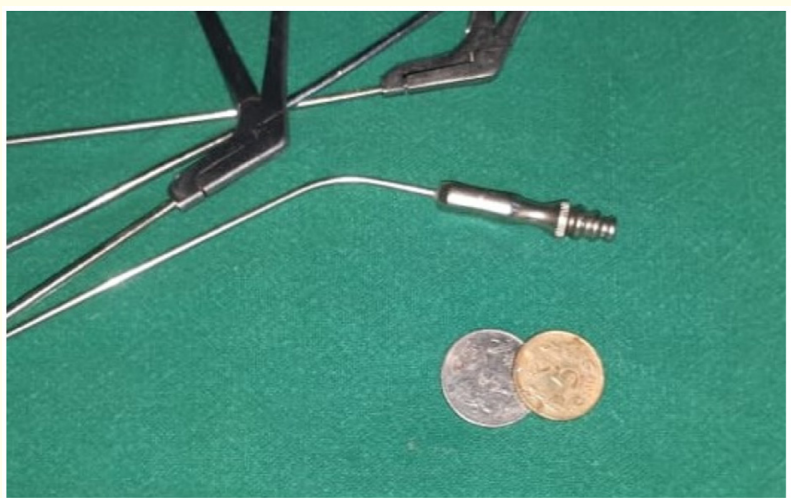

Figure 3: Intra-operative picture shows two different types of coin removed from cricopharynx.

\section{Discussion}

In the pediatric age group the coin is the most common foreign body swallowed. But ingestion of multiple coins is a rare entity. Coins generally get lodged below the cricopharyngeus muscle (upper esophagus) and lie in the coronal plane [5]. Once the foreign body has passed this upper esophageal constriction it crosses the alimentary tract without any significant problem [5].

Removal of the foreign body is mandatory as it may lead to life threatening complications of esophageal perforation, aspiration and mediastinitis [5]. Foreign body impacted in upper esophagus may be removed with variety of techniques like rigid esophagoscopy, using inflated Foley's catheter for proximal dislodgement [6], Magill's forceps used along with direct laryngoscope [7] and flexible fiberoptic esophagoscopy. The rigid esophagoscopy, which is mostly being followed, is considered the gold standard [8].

Whenever a radio-opaque foreign body is suspected, plain $\mathrm{x}$ rays are asked for as the first step. The x-ray tells the type of the object, its shape, size, location and also gives an idea of any possible complication, if it has already occurred. Usually, the neck xray (posterior-anterior and lateral view) is adequate, but if there is suspicion of migration distally in the gastrointestinal tract, the abdominal x-rays may be done. Flat circular objects like coins, bottle caps, or button batteries usually lie oriented in the coronal plane in the esophagus (as the esophagus is dorso-ventrally flattened) and thus cast a round shadow on the posterior- anterior view. But 
a similar object when aspirated into trachea, will lie oriented in the sagittal plane and thus cast a round shadow on the lateral view. Thus, always both views are asked for pre-operatively.

Further a circular "coin-like" object when visible on x-ray, needs careful review to rule out a "halo" or "double-ring" appearance which is seen in a button battery. This may need early intervention to avoid its leak and necrosis of surrounding tissue. Also, like in our case, it may be due to multiple coin ingestion. The chest $\mathrm{x}$-ray has high sensitivity, specificity and accuracy in differentiating the coin from button battery [9]. Multiple coins tend to stick together because of the surface tension and the two-dimensional projection on x-ray film often fails to indicate their presence.

Similarly, in our case the 2 coins gave a single circular shadow in the AP view due to overlapping. It was on lateral view, because of the difference in the height of the anterior and posterior border that a doubt of multiple coins arose, which was confirmed in revised history. It is important to have this in mind thus comprehensive history and detailed examination of AP and lateral view x-rays is necessary [10].

\section{Conclusion}

Multiple foreign bodies being swallowed in the pediatric population, as in our case, is quite rare. A thorough history and detailed radiological examination helped in preoperative confirmation of the same. Intra-operative care avoided unwanted complications like, recurrence of symptoms (as would have occurred after removal of one coin) and legal implications.

\section{Bibliography}

1. Bhasin A and Elitsur Y. "Esophageal stenosis, a rare complication of coin ingestion: Case report". Gastrointestinal Endoscopy 59.1 (2004): 152-154.

2. Janik JE and Janik JS. "Magill forceps extraction of upper esophageal coins". Journal of Pediatric Surgery 38.2 (2003): 227-229.

3. Lai AT., et al. "Risk factors predicting the development of complications after foreign body ingestion". British Journal of Surgery 90.12 (2003): 1531-1535.
4. Lemberg PS., et al. "Aerodigestive tract foreign bodies in the older child and adolescent". Annals of Otology, Rhinology, and Laryngology 105.4 (1996): 267-271.

5. Amin MR., et al. "Predicting outcome in pediatric coin ingestion". International Journal of Pediatric Otorhinolaryngology 59.3 (2001): 201-206.

6. Campbell JB and Condon VR. "Catheter removal of blunt esophageal foreign bodies in children. Survey of the Society for Pediatric Radiology". Pediatric Radiology 19.6-7 (1989): 361-365.

7. Mahafza TM. "Extracting coins from the upper end of the esophagus using a Magill forceps technique". International Journal of Pediatric Otorhinolaryngology 62.1 (2002): 37-39.

8. Bending DW. "Removal of blunt esophageal foreign bodies by flexible endoscopy without general anesthesia". American Journal of Diseases of Children 140 (1986): 789-790.

9. Malik SA., et al. "Diagnostic Accuracy Of Plain X-Ray Lateral Neck In The Diagnosis Of Cervical Esophageal Foreign Bodies Keeping Oesophagoscopy As Gold Standard". Journal of Ayub Medical College Abbottabad 30.3 (2018): 386-388.

10. Whelan-Johnson S and Hall CE. "Multiple oesophageal foreign bodies: the importance of two radiological views". Journal of Laryngology and Otology 123.1 (2009): 121-122.

\section{Volume 3 Issue 11 November 2021}

(C) All rights are reserved by Manish Gupta., et al. 